3 Lauren Y. Cheng ${ }^{1}$, Peng Dai ${ }^{1}$, Lucia R. Wu ${ }^{1}$, David Yu Zhang ${ }^{1,2, *}$

4

5 1. Department of Bioengineering, Rice University, Houston, TX 77030, USA

2. Systems, Synthetic, and Physical Biology, Rice University, Houston, TX 77030, USA

$7 \quad *$ genomic.dave@gmail.com

Abstract

10 Direct capture and sequencing revealed a new DNA population in biofluids, named ultra-short

11 single-stranded DNA (ussDNA), which was neglected by conventional DNA extraction and

12 sequencing approaches. Evaluation of the size distribution and abundance of ussDNA in biofluids

13 manifested generality of its presence in human, animal, and plants. Red blood cells were found

14 to contain abundant ussDNA with enriched functional elements, and thus possesses great

15 potential as a novel DNA biomarker.

\title{
17 Main
}

18 To date, liquid biopsy predominantly focuses on cell-free DNA (cfDNA), which has a prominent

19 peak at $167 \mathrm{bp}$, representing the size of double-stranded DNA (dsDNA) chains wrapped around a

20 nucleosome unit ${ }^{1,2}$. CfDNA and tissue biopsy testing have established high concordance in

21 advanced cancers ${ }^{3,4}$; however, cfDNA concentration is low, especially in non-cancer individuals ${ }^{5}$,

$22{ }^{6}$, limiting its application outside of oncology. Moreover, recent studies on cfDNA demonstrate 
23 that circulating tumor DNA (ctDNA), the tumor-derived portion of cfDNA, are shorter compared

24 to $\mathrm{cfDNA}^{7,8}$. Another study shows that single-stranded library preparation method has superior

25 cfDNA yield, especially in sub-nucleosomal sizes ${ }^{1}$. These findings suggest that circulating DNA

26 may exist at sizes below nucleosomal size and are likely single-stranded or partially single-

27 stranded. However, conventional DNA extraction methods based on spin column or Solid Phase

28 Reversible Immobilization (SPRI) are susceptible to high loss at sizes below 100nt ${ }^{9}$, 10. Thus, a

29 method tailored for extraction of ultra-short single-stranded DNA (ussDNA) is necessary to uncover the sub-nucleosomal space and explore the presence of biomarkers in various biofluids.

Here, we present a method that characterizes ussDNA from biofluids by direct capture and sequencing, in which probe captured ussDNA are prepared for next generation sequencing (NGS)

Biotin-3'), and the rationale is that 10-mer degenerate bases bear $\sim 1$ million unique sequences,

37 which can be largely matched to the massively diverse human genome. LNA bases can enhance duplex stability and thus lower melting temperature ${ }^{11}$, so the probe design enables efficient hybridization of diverse populations of ussDNA at room temperature in high salinity buffer condition. The single-stranded library preparation approach leverages a splinter adapter ligation

41 step optimized for recovery of short fragments using organic solvent ${ }^{12}$. Sequencing of 24 different

42 synthetic ssDNA oligos with sizes from 20nt to 70nt showed that all strands were recovered at

43 their expected sizes (Fig. S1a-b), albeit 100-fold yield variation was observed following capture

44 (Fig. S1c-d), which could be partially explained by highly varied hybridization rates among 
45 different sequences ${ }^{13}$. The method demonstrated $9 x$ yield for ssDNA compared to dsDNA as

46 manifested by reads retrieved from equal molarity spike-in (Fig. S1h). In addition, an adapter

47 dimer blocker was designed according to a published blocker displacement amplification

48 principle ${ }^{14}$ to reduce dimerized adapters in library (Fig. S1f-g).

50 Next, the direct capture and sequencing approach was applied to plasma, peripheral blood

51 mononuclear cell (PBMC) and red blood cell (RBC) separated from total blood and ussDNAs were

52 found in all blood fractions. RBC-derived ussDNA displayed wide distribution from 20nt to larger

53 than 200nt, with the highest concentration appeared at 70nt (Fig. 1b); ussDNA from plasma

54 showed a sharp peak at 50nt, and the nucleosome-sized cfDNA was also recovered, likely

55 captured via partial single-strands in dsDNA (Fig. 1c); PBMC-derived ussDNA had a similar 50nt

56 peak as plasma ussDNA (Fig. 1d). RBC in this healthy individual was found to be abundant in

57 ussDNA, whose concentration is approximately 10-fold higher than plasma, and comparable to

58 PBMC. This finding is counterintuitive because human RBCs are nucleus-free and thus are

59 expected to have low DNA content. Note that when probes were absent from the system, ssDNA

60 spike-in strands were not recuperated in RBC or plasma library, and the 50nt mode in plasma

61 ussDNA did not appear (Fig. S1i-j), implying that probes are effective in capturing ssDNA. UssDNA

62 in both RBC and plasma exhibited dominant peaks at 50nt after dsDNase treatment that is

63 specific to dsDNA, and distributions shifted to no-template-control following DNase I digestion,

64 suggesting DNA material with single-strandness at 50nt (Fig. S2a-d).

65 
66 To quantitate the concentration of blood ussDNA, ssDNA oligos were spiked into each blood

67 fraction (Fig. S3a) at $1 \mathrm{pM} /$ strand and the concentration was estimated from the relative

68 abundance of ussDNA and reference sequences. The estimation was validated by curve fitting of

69 varied spike-in concentrations and the corresponding reads fraction of spike-in sequences (Fig.

70 S3b-c). In the small cohort of 17 healthy individuals, the approximated ratio of the mean of

71 ussDNA concentration is PBMC:RBC:plasma = 27:7:1 (Fig. 2a). Both RBC and PBMC contain

72 significantly more ussDNA than plasma $(p<0.0001)$, despite higher variations in concentration.

73 UssDNA concentrations in different blood fractions had no apparent relationship as RBC ussDNA

74 concentration merely displayed slightly positive correlation with its concentration in plasma and

75 PBMC (Fig. 2h, Fig. S4f). Correlation between ussDNA concentration and age was investigated

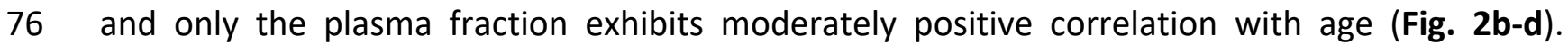

77 UssDNA concentration in RBC is higher in female than male individuals $(p<0.05)$, whereas no

78 gender difference is manifest in plasma or PBMC counterparts (Fig. 2e-g). When spike-in strands

79 were added to total blood prior to fractionation (Fig. S3d), the reference strands retained

80 primarily in aqueous phase, i.e., the plasma fraction, and were depleted in cellular phases,

81 especially after washing (Fig. S3g-k), inferring that association of bare reference strands with

82 cellular components is not intrinsically favored. In contrast, PBS washing did not deprive RBC of

83 its ussDNA content (Fig. S3e-f), suggesting the association of RBC ussDNA with cellular structures.

85 We then sought to understand whether ussDNAs originate from entire or parts of the human

86 genome. Whole genome alignment revealed global distribution in autosomes and chromosome

$87 \mathrm{X}$ from RBC-derived ussDNA (Fig. 1e). RBC ussDNA distributed uniformly among all chromosomes 
(Fig. S4b). However, 2880-fold enrichment of mitochondria genome was observed in RBC ussDNA,

89 implying exogenous source of ussDNA in RBC fraction because erythrocytes have no

91 compared to nuclear ussDNA (Fig. S4c). Although enrichment of mitochondrial cfDNA was

92 reported 6,15 , the fold enrichment might be partially explained by the fact that human cells

93 contain 200-4000 copies of mitochondria dependent on metabolic intensity ${ }^{16}$. With regards to

94 focal distribution of functional elements, their fractions in reads were compared with

95 corresponding genome fractions. We assumed that the reads fraction is representative of their

96 biological distribution in ussDNA because for highly diverse sequences the biases in hybridization,

97 PCR amplification, or random sampling become negligible. RBC ussDNA showed significant

98 enrichment in gene coding regions and enhancer elements $(p<0.0001)$, as well as depletion in

99 intron and telomere regions $(\mathrm{p}<0.0001)$ compared to their distributions in genome (Fig. $\mathbf{2} \mathrm{i}-\mathbf{j})$. The

100 enriched fraction of coding or regulatory regions in RBC ussDNA, in combination with the

101 transporting role of RBCs, may suggest functional roles of RBC ussDNA. Like RBC-derived ussDNA,

102 plasma ussDNA also presented uniformity among all chromosomes despite low coverage owing

103 to lower sequencing depth (Fig. S4a,d). Mitochondrial ussDNA in plasma was enriched 430-fold

104 and displayed similar size distribution to its nuclear counterpart (Fig. S4e). In plasma ussDNA,

105 depleted intron and telomere regions $(p<0.0001)$ and enriched enhancers $(p<0.01)$ were also

106 observed (Fig. S4g-h).

108 Last, we expanded our study to biofluids of other animal species and plants to investigate the 109 generality of ussDNA. Unlike RBC ussDNA from human where its distribution is smooth, ussDNA 
110 from bovine and pig RBC displayed spiky peaks from 30nt to 70nt with 10nt periodicity followed

111 by a smooth decay (Fig. S5a,d). UssDNA from bovine plasma showed a similar peak to its human

112 counterpart, except that the peak centered at 63nt instead of 50nt (Fig. S5b). Plasmas from pig

113 and rabbit, however, had decreasing oscillations of 10nt periodicity from 30nt to above 100nt

114 (Fig. S5e,g). The 10nt periodicity is representative of a helical turn around the nucleosome core, 115 and infers the generation mechanism via DNase I cleavage ${ }^{1}$. Interestingly, the periodic pattern 116 appeared in PBMC of pig (Fig. S5f) and milk of bovine (Fig. S5h) but not in PBMC of bovine (Fig.

117 S5c). To confirm whether animal ussDNA was free of human DNA contamination, bovine ussDNA

118 was cross-aligned to human reference genome and notably reduced coverage was found (Fig.

119 S6a-d). Plant-derived ussDNA showed varied sizes and concentrations. In kiwifruit, ussDNA

120 peaked at 34nt followed by gradual reduction (Fig. S7a). In orange and cherry, ussDNA spikes

121 between 20-30nt and short artifacts that appeared in all libraries are indistinguishable, and their 122 concentrations above 30nt are low, especially in cherry (Fig. S7b-c).

124 In summary, we present a method tailored to investigate the understudied sub-nucleosomal 125 ussDNA in biofluids. We found ussDNA in blood fractions of human as well as animal species, and 126 plant-based biofluids with different quantities and size distributions. To our surprise, human RBC 127 is rich in ussDNA, which challenges the notion that human RBC do not contain DNA. Enrichment 128 of RBC ussDNA in mitochondrial genome and functional regions such as gene coding region and 129 enhancer elements may imply functional roles of RBC ussDNA. Collectively, our finding may direct 130 to a promising biomarker that has high concentration and won't subject to interference from 131 nucleus DNA. This study points for the first time to a new population of circulating DNA, yet little 
132 is known other than its size representation and concentration, and thus future work is awaiting

133 to elucidate its generation mechanism, tissue-of-origin, disease indications, etc.

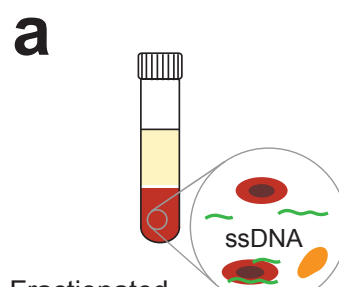

Fractionated Blood
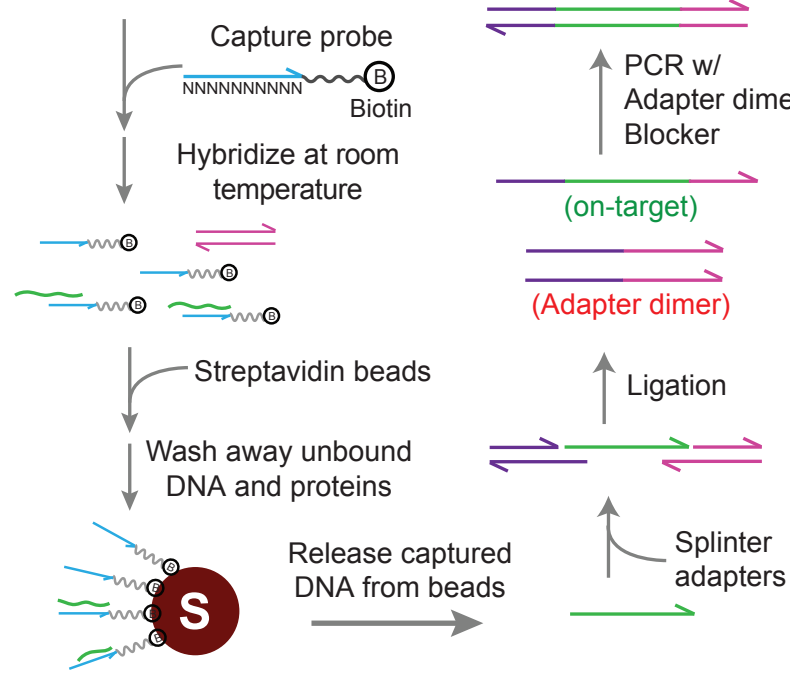

\section{e}

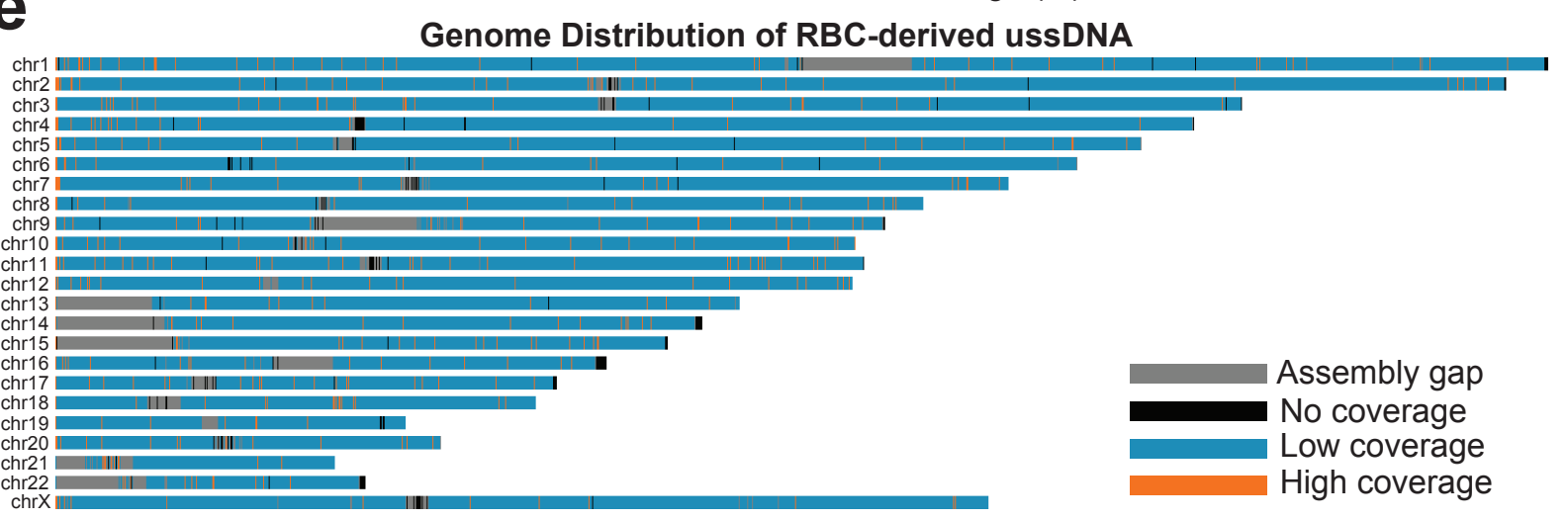


138 Figure 1: Direct capture and sequencing discovered ussDNA in blood components. (a) Direct

139 capture and sequencing workflow. ussDNAs captured by LNA probes were separated from

140 biofluids on streptavidin beads. Extracted ussDNAs were then ligated with adapter sequences

141 and prepared for sequencing. (b-d) Length distribution of ussDNA found in (b) RBC (c) plasma

142 and (d) PBMC. Peaks below 20nt that appeared in all samples are artifacts produced by the

143 workflow. Magenta spikes represent ssDNA spike-in references, 4 different strands were spiked

144 in at each size at concentration of 1pM each. (e) Human genome distribution of RBC-derived

145 ussDNA in autosomes and chromosome $X$. Regions where no reads aligned to were shown in

146 black; gaps in human genome assembly GRCh38 were colored in grey. Regions with more than

$1472 x$ global density were colored in orange. 
a

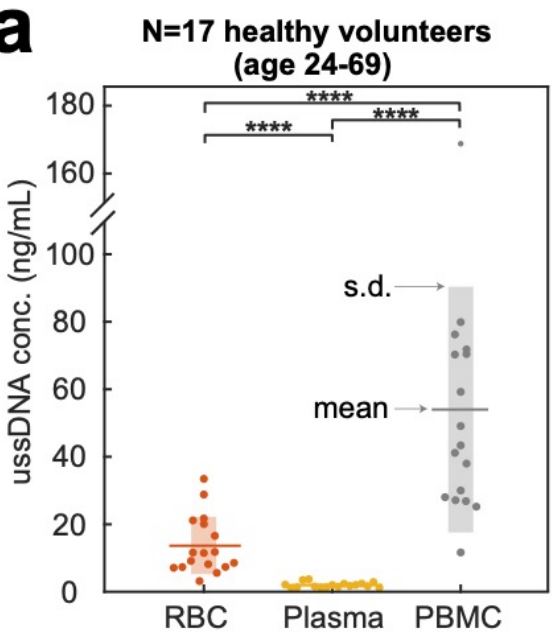

b
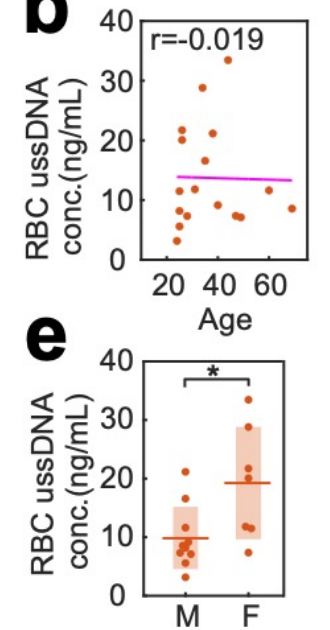
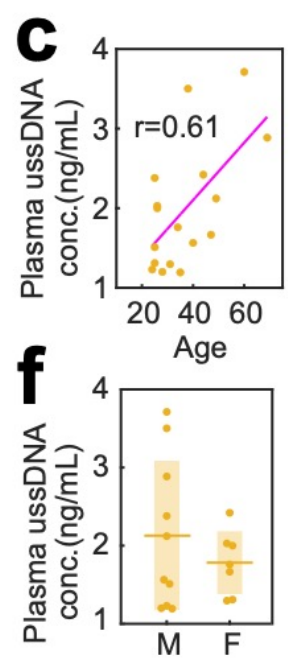

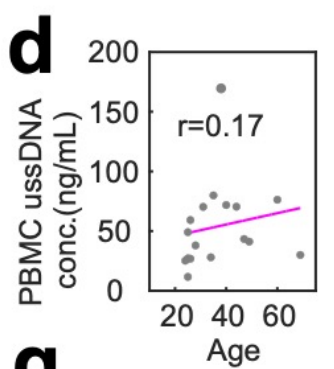

g

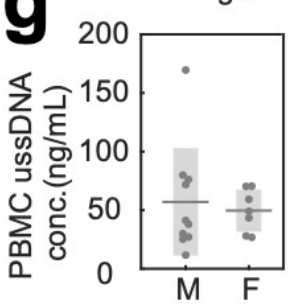

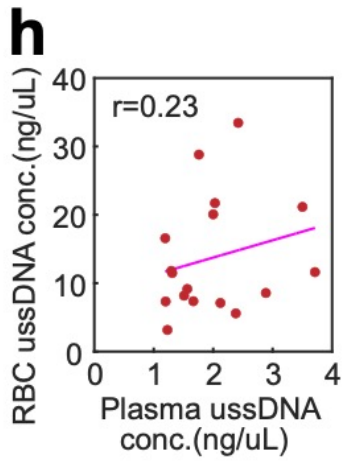
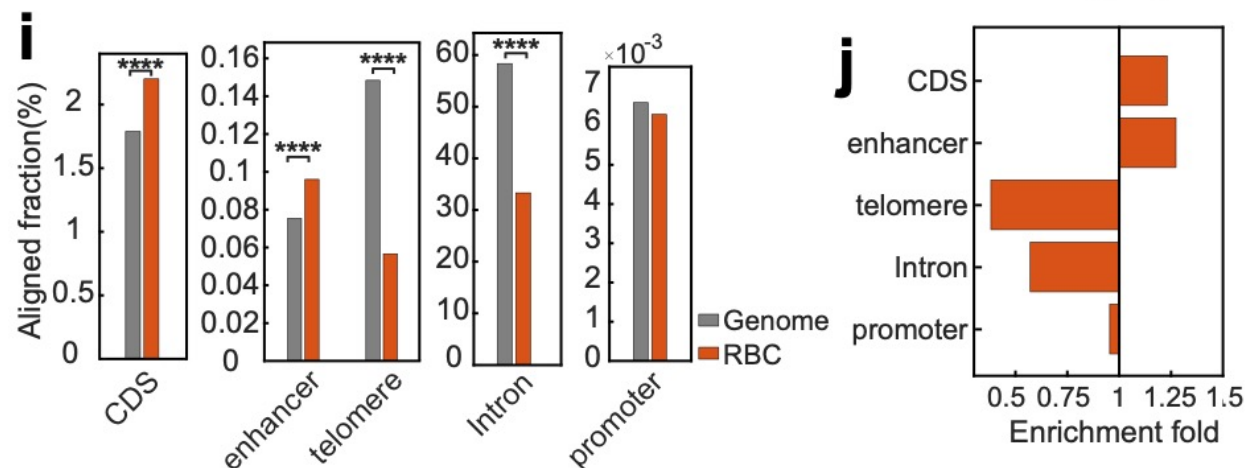

Figure 2: Quantification and focal genome distribution of blood ussDNA. (a)ussDNA

151

152

153

154

155

156

157

158

159

concentrations in RBC, plasma and PBMC fractions from healthy volunteers. Boxplot represents

standard deviation from the mean. ussDNA concentration's rank is PBMC > RBC > plasma (paired

t-test, $* * * * \mathrm{p}<0.0001)$. (b-d) Correlation of age and concentrations of ussDNA in (b) RBC, (c)

plasma and (d) PBMC. Only plasma ussDNA concentration exhibits moderately positive correlation with age from Pearson's correlation coefficient r. (e-g) ussDNA concentrations grouped by gender ( $M$ = male, $F=$ female) in (e) RBC, (f) plasma and (g) PBMC. Welch's t-test shows significantly higher plasma ussDNA concentration in females compared to males $(* p<0.05)$.

(h) Correlation between RBC ussDNA concentration and plasma ussDNA concentration. (i) Fraction of RBC ussDNA aligned to functional elements compared to their fractions in human 
161 depletion in telomere and intron regions are observed in RBC ussDNA compared to random
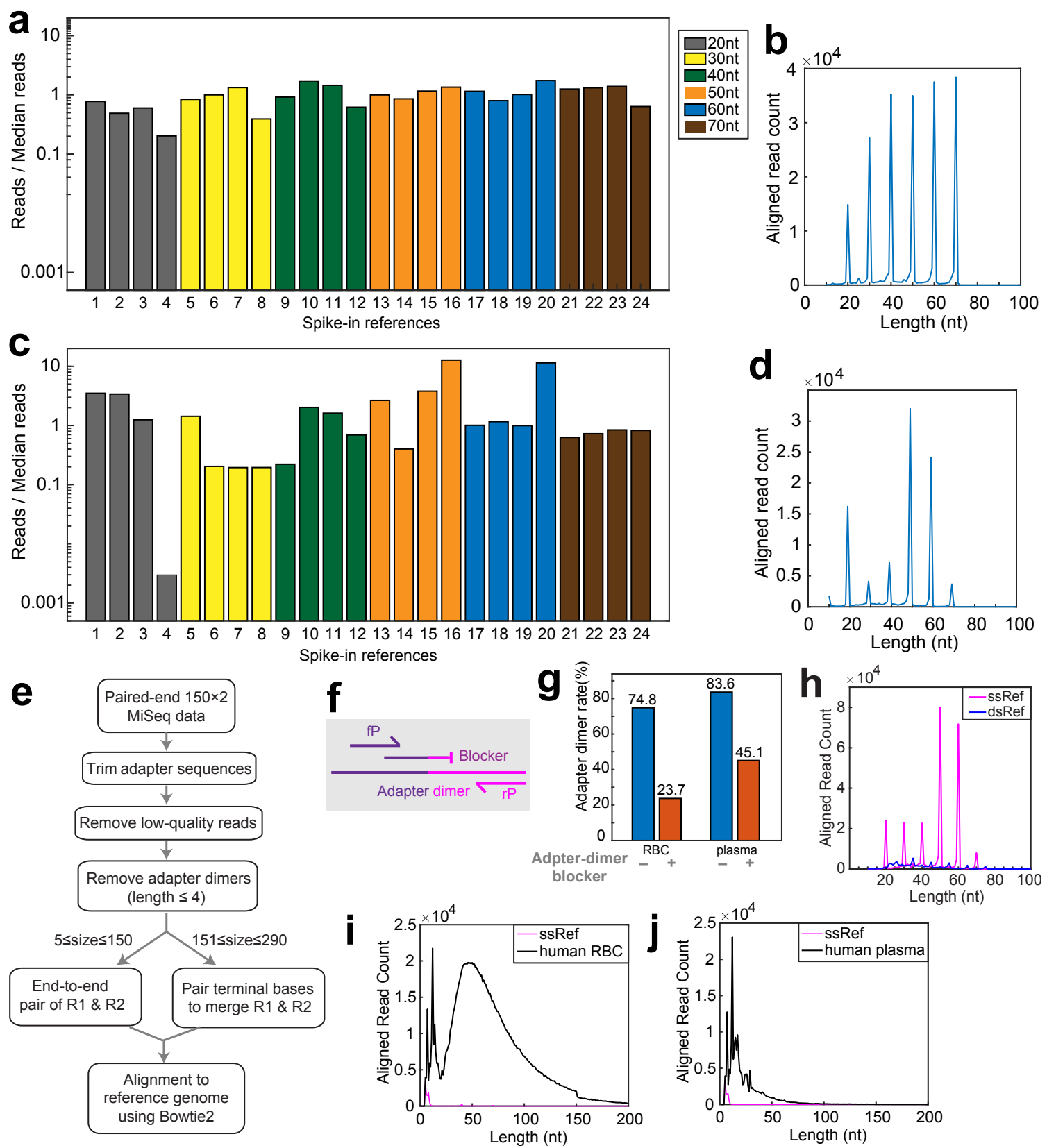
166 Figure S1: Workflow characterization. (a) Fold difference of reads aligned to each 1pM spike-in

167 reference after library preparation using SRSLY PicoPlus kit. Reference strands are sSDNA of 168 lengths from $20 n$ to 70 nt with 10 t interval. 23/24 references are within 2 -fold from the median 169 reads. Length of reference ssDNA are color coded in the bar graph. (b) Length distribution of 170 library prepared from spike-in ssDNA reference. $88.93 \%$ of aligned reads were recovered at 171 expected sizes. (c) Fold difference of reads aligned to each 1pM spike-in reference after ussDNA 172 capture and library preparation using SRSLY PicoPlus kit. All reference materials are recovered in 173 the sequencing library, albeit with reduced reads uniformity, suggesting variations in capture 174 yield. 22/24 references are within 10-fold from the median reads. (d) Length distribution of 175 library prepared from captured spike-in ssDNA reference. Sharp peaks at expected sizes suggest 176 unbiased length representation, and $86.26 \%$ of aligned reads are at expected sizes. (e) 177 Bioinformatics pipeline for sequencing data analysis. (f) Design schema of adapter dimer blocker. 178 Purple lines: i5 adapter sequences, pink lines: i7 adapter sequences, fP: forward primer, rP: 179 reverse primer. (g) Adapter dimer rate of libraries prepared from RBC or plasma with (orange 180 bars) and without (blue bars) adapter dimer blocker. (h) Reads aligned to ssDNA (magenta) and 181 dsDNA (blue) references at equal molarity of 1pM/strand. dsDNA references are sized at 25:10:75 182 nt with 4 different strands at each size. 9x more reads are aligned to ssDNA reference than dsDNA 183 reference, suggesting capture preference for ssDNA. (i-j) Length distribution of ussDNA in (i) RBC 184 and (j) plasma from no-probe capture. Experiments were performed following standard direct 185 capture and sequencing workflow except that LNA probes were not added. 
a
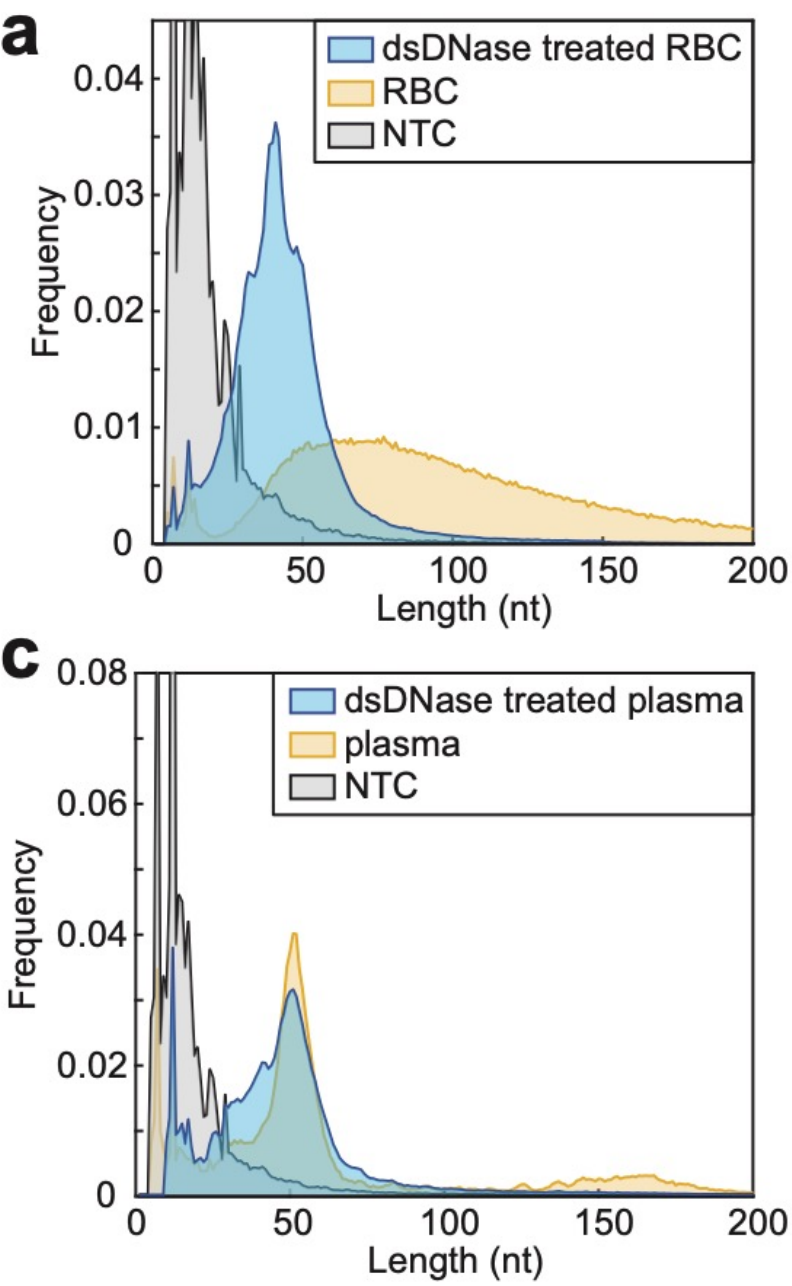
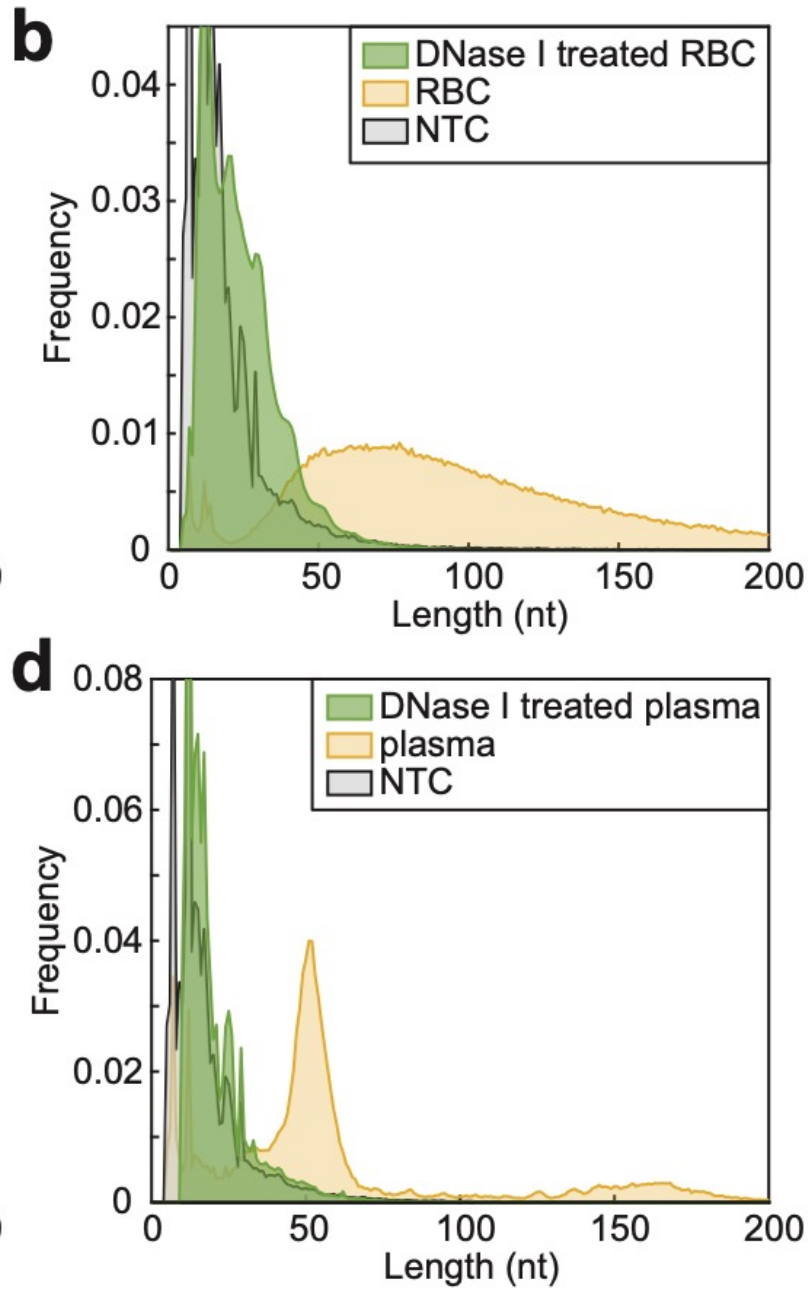

188 Figure S2: Blood-derived ussDNA underwent enzymatic digestion. Length distribution of (a)

189 dsDNase treated RBC ussDNA, (b) DNase I treated RBC ussDNA, (c) dsDNase treated plasma

190 ussDNA and (d) DNase I treated plasma ussDNA compared with untreated RBC or plasma and no

191 template control (NTC). Yellow shades: untreated ussDNA in RBC or plasma; grey shades: NTC;

192 Blue shades: ussDNA in RBC or plasma treated by dsDNase; Green shades: ussDNA in RBC or

193 plasma treated by DNase I. dsDNase specifically digests double-stranded DNA and DNase I

194 cleaves all DNA structures into short fragments. 


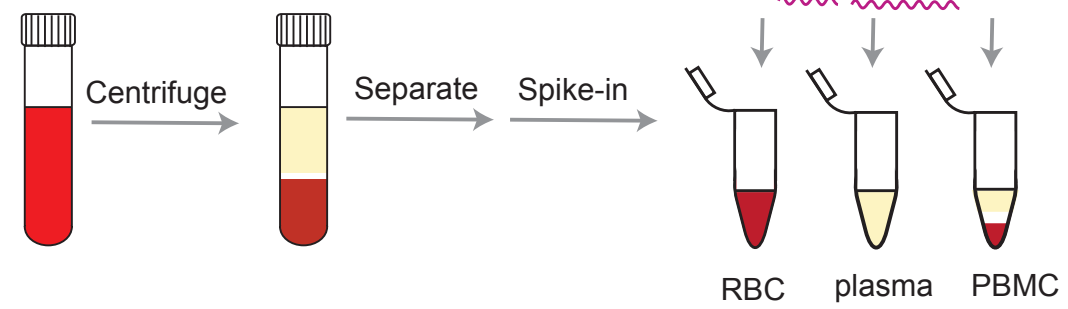

b

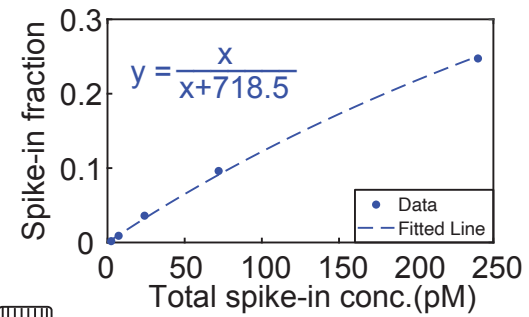

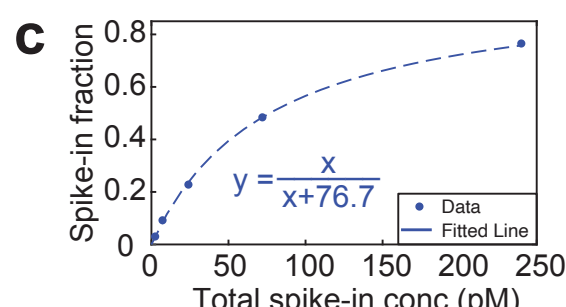
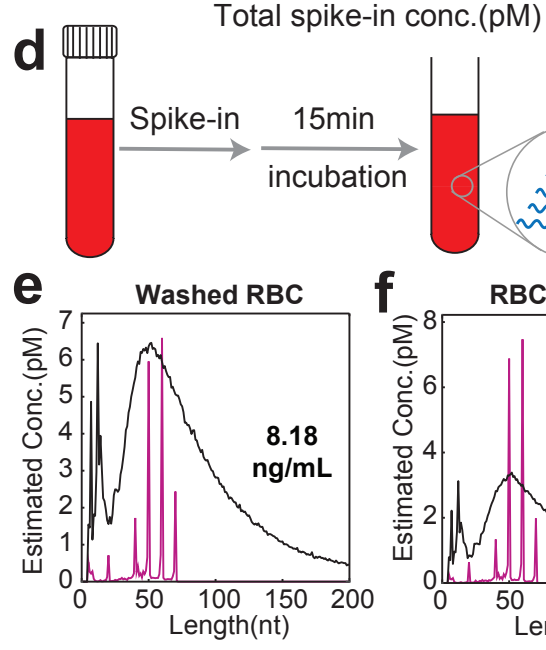

Total spike-in conc.(pM)

\section{7}
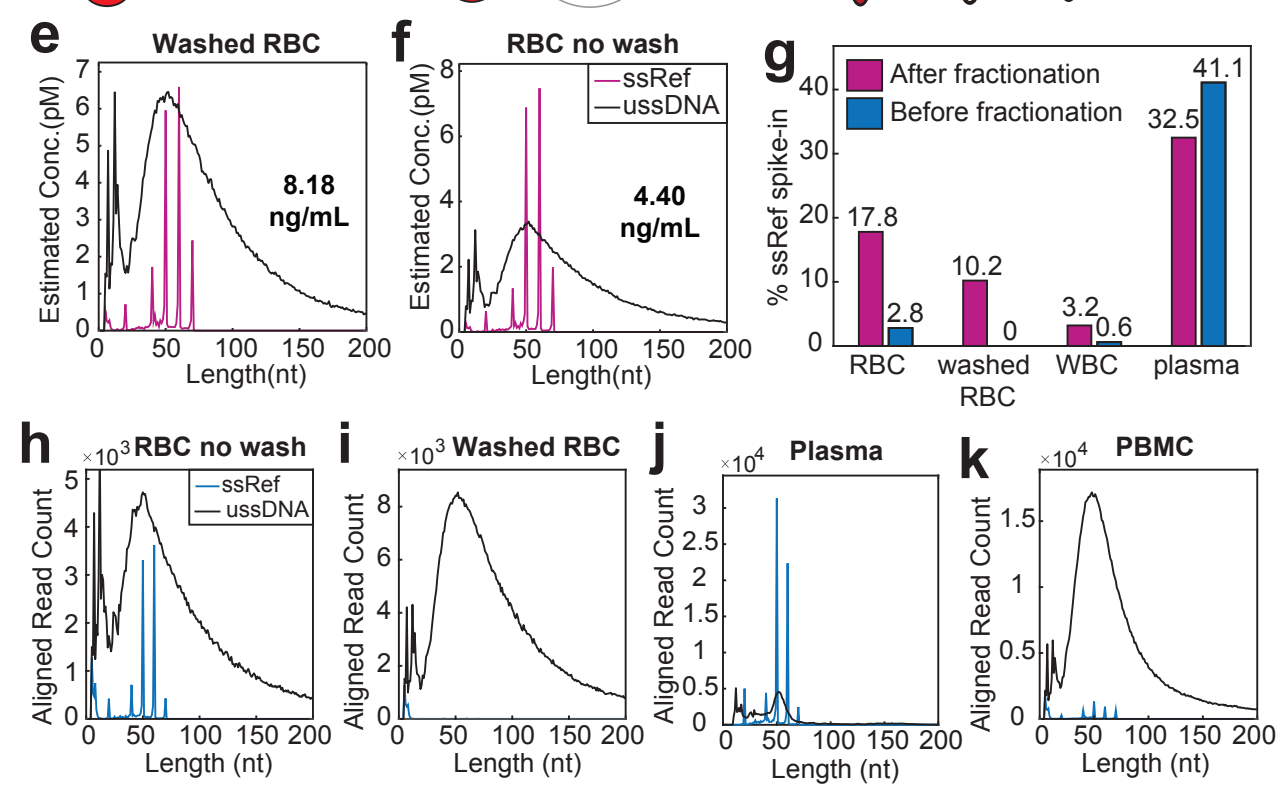

Figure S3: Spike-in of short synthetic DNA oligos as reference. (a) Schematic workflow of spiking

in ssDNA strands to fractionated blood for quantitating blood-derived ussDNA. ssDNA references

are composed of 24 strands at sizes 20:10:70 nt with 4 different strands at each size. (b-c)

Calibration of spike-in fraction vs. total spike-in concentration to model ussDNA concentration in

(b) RBC and (c) plasma. ssDNA references were spiked in at $0.1 \mathrm{pM}, 0.3 \mathrm{pM}, 1 \mathrm{pM}, 3 \mathrm{pM}$ and 10pM 
202 per strand and 24 strands in total. Dashed line stands for curve fitting of spike-in data points to y

$203=x /(x+k)$ where $y=$ spike-in fraction, $x=$ total spike-in concentration $(p M)$ and $k$ is fitting

204 coefficient representing ussDNA concentration in pM. The estimated ussDNA molarity is

$205718.5 \mathrm{pM}$ in RBC and 76.7pM in plasma. (d) Schematic workflow of spiking in ssDNA strands to

206 total blood followed by fractionation. (e-f) Length distribution and concentration of ussDNA in (e)

207 washed RBC (RBC pellet washed twice in PBS buffer) and (f) non-washed RBC. (g) Comparison of

208 spike-in fraction between ssDNA strands added before (blue bars) and after (magenta bars)

209 whole blood was fractionated. (h-k) Length distribution of ussDNA and ssDNA reference in blood

210 fractionated after whole blood spike-in. Whole blood was fractionated into (h) RBC (i) washed

211 RBC (j) plasma and (k) PBMC. 
a

Genome Distribution of plasma-derived ussDNA

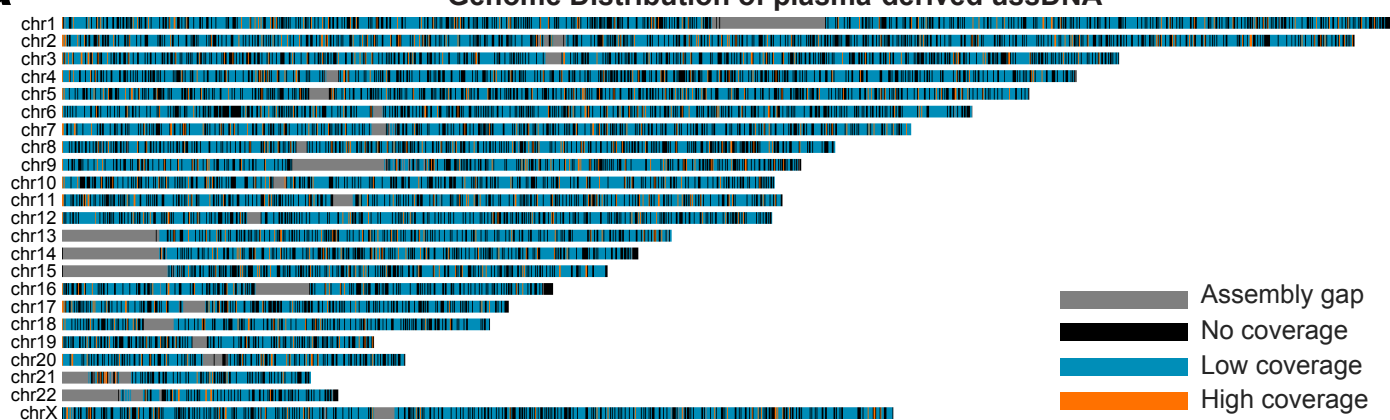

b

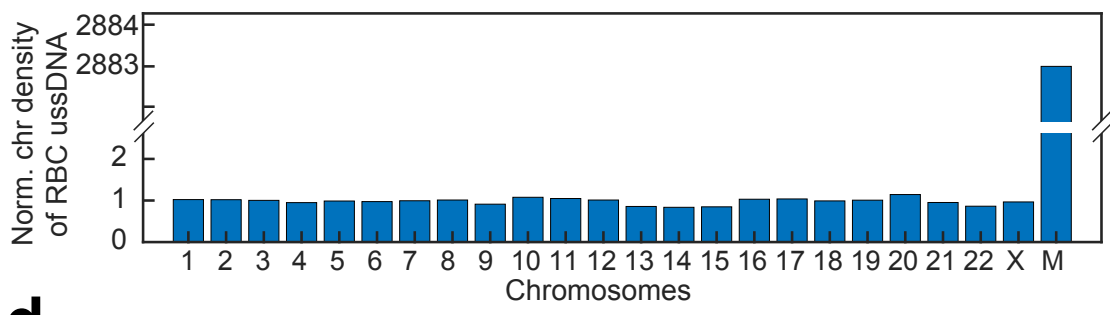

d
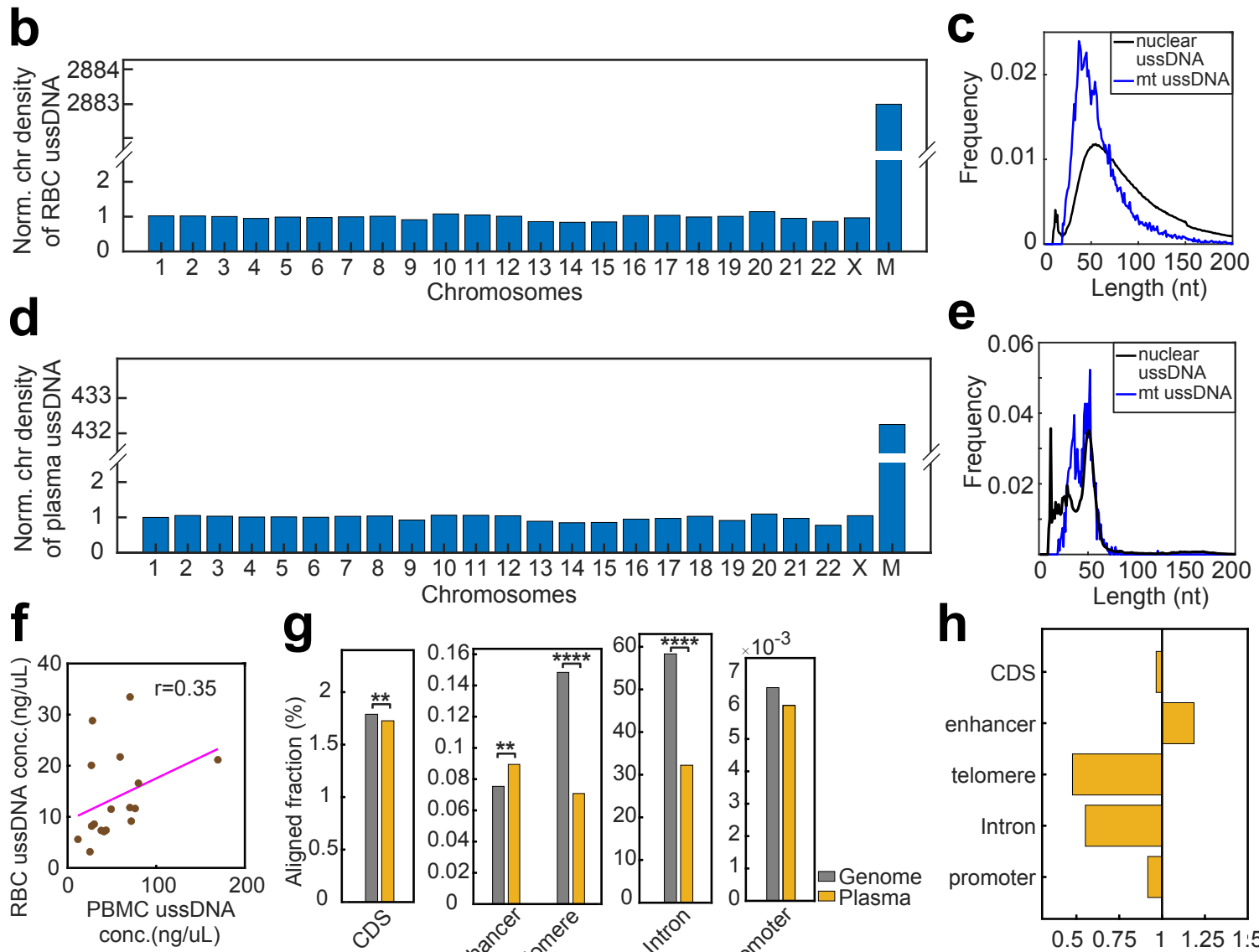

\section{Chromosomes}
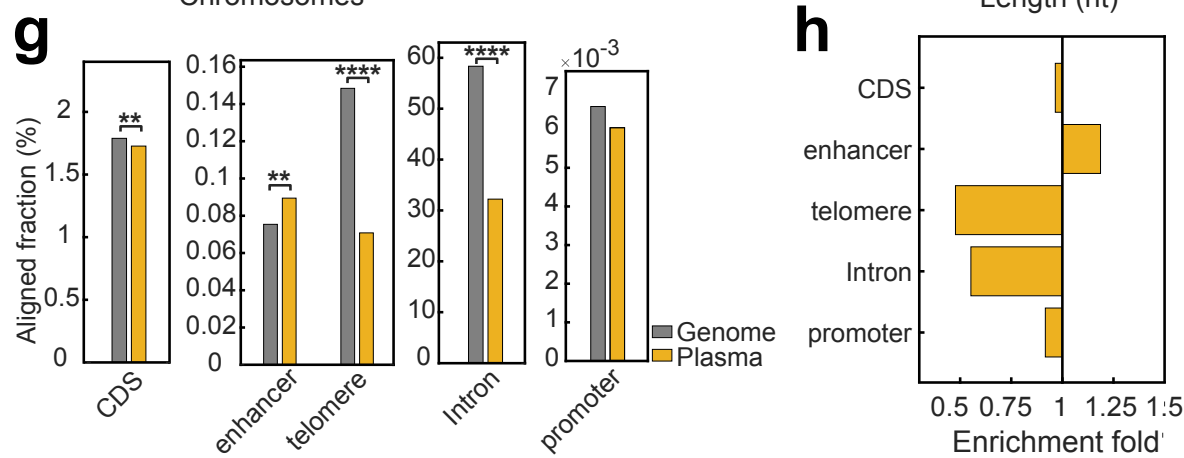

214 Figure S4: Genome distribution of blood-derived ussDNA. (a) Human genome distribution of

215 plasma-derived ussDNA in autosomes and chromosome $\mathrm{X}$. Regions where no reads aligned to

216 were shown in black; gaps in human genome assembly GRCh38 were colored in grey. Regions

217 with more than $2 x$ global density were colored in orange. (b) RBC ussDNA density in

218 chromosomes (including mitochondria genome, ChrM) normalized to global density. (c) Size 
219 distribution of mitochondrial ussDNA and nuclear ussDNA in RBC. (d) Plasma ussDNA density in

220 chromosomes normalized to global density. 2880-fold and 430-fold enrichment of mitochondria

221 genome are observed in RBC and plasma ussDNA, respectively. (e) Size distribution of

222 mitochondrial ussDNA and nuclear ussDNA in plasma. (f) Correlation between RBC ussDNA

223 concentration and PBMC ussDNA concentration. Pearson's correlation coefficient $(r)$ showed

224 weak positive correlation. (g) Fraction of plasma ussDNA aligned to functional elements

225 compared to their genome fractions. CDS: gene coding region. Plasma ussDNA exhibited

226 significant enrichment of enhancer, and depletion in telomere, CDS and intron regions compared

227 to random distribution ( $* * p<0.01, * * * * p<0.0001)$. (h) Fold enrichment of functional elements

228 in ussDNA derived from plasma. 

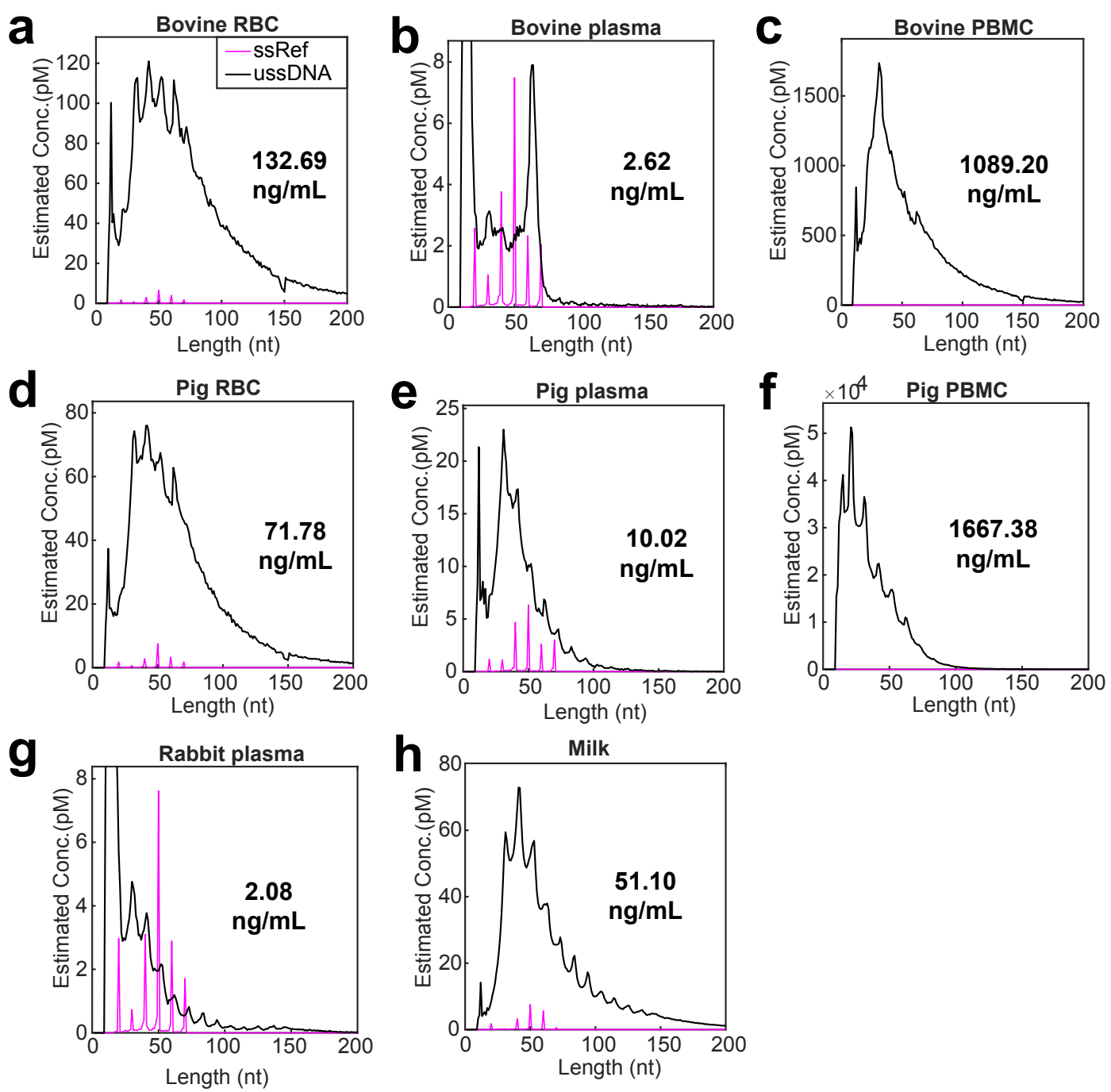

231 Figure S5: Size distributions and concentrations of ussDNA found in animal biofluids including

232 (a) bovine RBC, (b) bovine plasma, (c) bovine PBMC, (d) pig RBC, (e) pig plasma, (f) pig PBMC, (g)

233 rabbit plasma, and (h) milk. Magenta spikes represent ssDNA spike-in references, 4 different

234 strands were spiked in at each size at concentration of $1 \mathrm{pM}$ each. Peaks below 20nt appear in

235 most specimens and are considered artifacts produced by the workflow. 
a

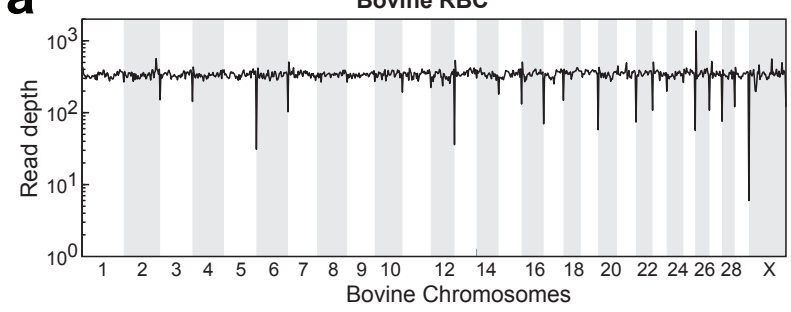

C

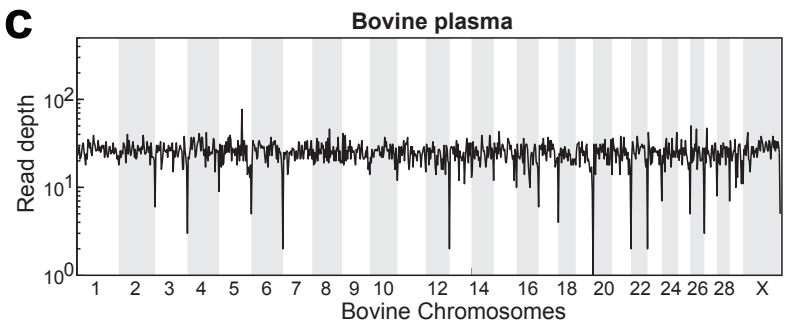

b
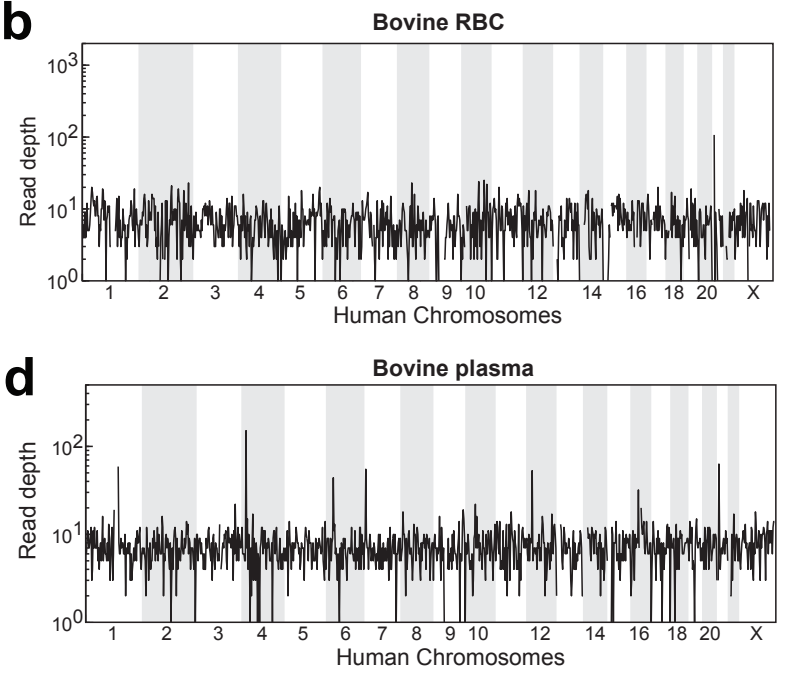

238 Figure S6: Cross-species genome alignment of bovine ussDNA. (a) Whole genome alignment of

239 bovine RBC ussDNA to bovine genome. (b) Cross genome alignment of bovine RBC ussDNA to

240 human genome. (c) Whole genome alignment of bovine plasma ussDNA to bovine genome. (d)

241 Cross genome alignment of bovine plasma ussDNA to human genome. Each data point on the

242 plotted line represents the number of reads aligned to 3 million nucleotide-sized bins. Cross-

243 aligning bovine ussDNA to human genome showed notably reduced coverage.

\section{4}

a

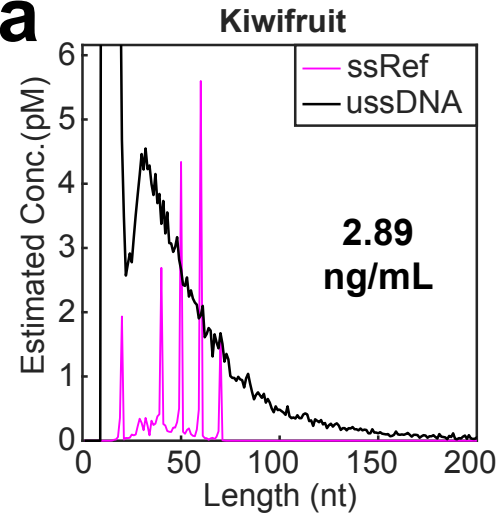

b

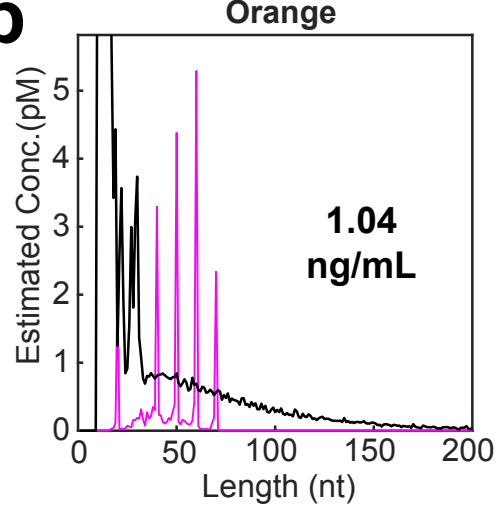

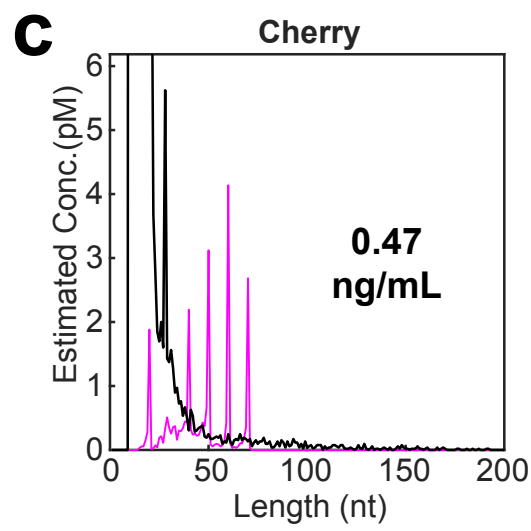


Figure S7: Size distributions and concentrations of ussDNA found in plant biofluids including (a)

247 kiwifruit juice, (b) orange juice, and (c) cherry juice. Magenta spikes represent ssDNA spike-in

248 references, 4 different strands were spiked in at each size at concentration of 1pM each. 


\section{Methods}

\section{Collection of biospecimen}

252 Total blood samples were collected into K2EDTA collection tubes (BD \#367863). N=7 of total

253 blood samples were collected from healthy volunteers by a certified phlebotomist through

254 venipuncture following IRB-FY2018-426 approved by Rice University. N=10 human blood samples

255 were purchased from ZenBio, shipped on collection day at $4^{\circ} \mathrm{C}$ and delivered next day.

256 Demographic information of blood donors was provided by ZenBio. Animal total blood samples

257 were purchased from Discovery Life Sciences, shipped on collection day at $4^{\circ} \mathrm{C}$ and delivered next

258 day. Demographic information of all 17 subjects was summarized in Table. S1.

260 Table. S1. Demographic information of healthy blood donors.

\begin{tabular}{|c|c|c|c|}
\hline No. & Collection type & Age & Gender \\
\hline 1 & On-site & 26 & Female \\
\hline 2 & On-site & 25 & Female \\
\hline 3 & On-site & 25 & Male \\
\hline 4 & On-site & 24 & Male \\
\hline 5 & On-site & 25 & Male \\
\hline 6 & On-site & 40 & Male \\
\hline 7 & On-site & 34 & Female \\
\hline 8 & ZenBio purchase & 60 & Male \\
\hline 9 & ZenBio purchase & 26 & Female \\
\hline 10 & ZenBio purchase & 38 & Male \\
\hline 11 & ZenBio purchase & 44 & Female \\
\hline 12 & ZenBio purchase & 31 & Female \\
\hline 13 & ZenBio purchase & 47 & Female \\
\hline 14 & ZenBio purchase & 28 & Male \\
\hline 15 & ZenBio purchase & 69 & Male \\
\hline 16 & ZenBio purchase & 49 & Male \\
\hline 17 & ZenBio purchase & 35 & Male \\
\hline
\end{tabular}


262 Milk was sampled from whole milk bought from grocery. Fruit juiced were collected via peeling

263 and squeezing the fruits. Centrifugation at $1500 x g$ for $10 \mathrm{~min}$ was performed and only

264 supernatant was collected.

265

266 Blood fractionation

267 Within 2 hours following blood drawing from volunteers or upon receive of purchased blood

268 samples, total blood samples were fractionated into plasma, peripheral blood mononuclear cell

269 (PBMC) and red blood cell (RBC) fractions as follows. First, the total blood was centrifuged at

$2701800 \mathrm{xg}$ for $15 \mathrm{~min}$ with brake set to $1 / 3$ of maximum level. Next, the upper clear layer of plasma

271 was separated without disturbing the interface, and a p1000 pipettor was used to carefully

272 collect PBMC layer to a new tube (*Residues of plasma and RBC remained in PBMC fraction

273 because buffy coat cannot be cleanly separated). Then for the remaining content of condensed

274 red blood cell pellet, $2 x$ volume of PBS was gently added and tube was inverted ten times for

275 mixing. The wash buffer was then removed following centrifuging at $500 \times \mathrm{g}$ for 5 minutes and

276 discarding the supernatant. The washing step was repeated and a final centrifugation at $1500 \mathrm{xg}$

277 for $5 \mathrm{~min}$ was performed. The washed RBC was collected by discarding supernatant and

278 transferring the pellet to a new tube. Unless otherwise mentioned, RBC fraction in this

279 manuscript refers to RBC following $2 x$ wash in PBS buffer.

\section{Direct capture}

282 Immediately following blood fractionation, $100 \mu \mathrm{L}$ of fractionated blood components were mixed

283 with LNA capture probe $\left(5^{\prime}-+\mathrm{N}+\mathrm{N}+\mathrm{N}+\mathrm{N}+\mathrm{N}+\mathrm{N}+\mathrm{N}+\mathrm{N}+\mathrm{N}+\mathrm{N} / \mathrm{Sp} 18 / \mathrm{Bio}^{-} 3^{\prime}\right.$, Integrated DNA 
284 Technologies) and hybrid capture buffer at final concentrations of $2 \mu \mathrm{M}$ capture probe, $0.5 \mathrm{M} \mathrm{NaCl}$,

$2851 \mathrm{x}$ TE and $0.1 \%$ tween 20 . The capture mixture was briefly vortexed and incubated at room

286 temperature for 2 hours with gentle shaking. For each sample, $60 \mu \mathrm{L}$ of Streptavidin beads

287 (Thermal Fisher, \#65001) that pre-equilibrated to room temperature were pelleted using a 288 magnetic rack and resuspended in $10 x$ volume of $0.5 \mathrm{M} \mathrm{NaCl}$ solution to wash the beads. Beads

289 were re-pelleted on the magnetic rack to remove the wash buffer and resuspended in $100 \mu \mathrm{L}$

290 buffer of $0.5 \mathrm{M} \mathrm{NaCl}, 1 \times \mathrm{TE}$ and $0.1 \%$ tween 20 . The buffer was removed by separating streptavidin

291 beads on a magnetic rack, and the pelleted beads were suspended in hybrid capture mixture and

292 incubated at room temperature for $30 \mathrm{~min}$ to allow binding of biotinylated probes to beads. The

293 streptavidin beads were then washed three times in $500 \mu \mathrm{L}$ of $0.5 \mathrm{M} \mathrm{NaCl}, 1 \mathrm{x} \mathrm{TE}$ and $0.1 \%$ tween20.

294 To collect bound DNA, beads were resuspended in $25 \mu \mathrm{L}$ of $0.1 \mathrm{x}$ TE buffer and heated at $95^{\circ} \mathrm{C}$ for

$2955 \mathrm{~min}$ to dissociate captured DNA from LNA probe. Eluant containing captured DNA was

296 transferred to a PCR tube following pelleting beads on magnetic rack.

\section{ussDNA library preparation}

299 Library preparation of captured single-stranded DNA was performed using SRSLY NanoPlus

300 (Claret Biosciences, \#CBS-K250B-96) according to manufacturer's instructions with modifications

301 to reduce adapter dimer. Specifically, $2 \mu \mathrm{L}$ of ss Enhancer and $18 \mu \mathrm{L}$ of eluted ssDNA were mixed

302 and heated at $98^{\circ} \mathrm{C}$ for $3 \mathrm{~min}$ and immediately cooled to $4^{\circ} \mathrm{C}$. Then $2 \mu \mathrm{L}$ of SRSLY NGS Adapter A,

$3032 \mu \mathrm{L}$ of SRSLY NGS Adapter B and $26 \mu \mathrm{L}$ of SRSLY Master Mix were added and the mixture was

304 incubated in a thermocycler at $37^{\circ} \mathrm{C}$ for $1 \mathrm{hr}$ with lid temperature set to $45^{\circ} \mathrm{C}$. The product was

305 purified with AMPure XP beads (Beckman Coulter, \#A63881) and isopropanol was added to 
306 increase recover of short fragments at ratio of [reaction product : AMPure beads : water :

307 isopropanol $=50 \mu \mathrm{L}: 59.4 \mu \mathrm{L}: 48.4 \mu \mathrm{L}: 11.6 \mu \mathrm{L}]$. Then the library was PCR-amplified with custom

308 designed PCR oligos (forward primer: 5'-ACACTCTTTCCCTACACGACG-3'; reverse primer: 5'-

309 GTGACTGGAGTTCAGACGTGT-3') and adapter dimer blocker (5'-

310 CACGACGCTCTTCCGATCTAGATCG/3SpC3/-3') that selectively suppress amplification of adapter

311 dimers. The PCR was performed with 400nM of each primer and $4 \mu \mathrm{M}$ of adapter dimer blocker

312 in PowerUp SYBR Green Master Mix (Thermal Fisher, \#A25742), and with thermocycle program

313 of initiation at $95^{\circ} \mathrm{C}$ for $3 \mathrm{~min}$ and 13 cycles of $95^{\circ} \mathrm{C}$ for $10 \mathrm{~s}$ and $60^{\circ} \mathrm{C}$ for 40 s (short as $95^{\circ} \mathrm{C} 3 \mathrm{~min}-$

$\left.314\left(95^{\circ} \mathrm{C} 10 \mathrm{~s}-60^{\circ} \mathrm{C} 40 \mathrm{~s}\right) \times 13\right)$. After purification with Monarch PCR \& DNA Cleanup kit (NEB, \#T1030S),

315 libraries were indexed using NEBNext Multiplex Oligos (NEB, \#E7780S) and Taq Universal probes

316 supermix (Bio-Rad, \#1725131) for 8 cycles of PCR following $95^{\circ} \mathrm{C} 3 \mathrm{~min}-\left(95^{\circ} \mathrm{C} 10 \mathrm{~s}-60^{\circ} \mathrm{C} 40 \mathrm{~s}\right) \times 8$.

317 The indexed library was purified with $1.5 x$ volume of AMPure beads and quality controlled by

318 bioanalyzer. Libraries were sequenced on an illumina Miseq or Nextseq instrument using 150x2

319 chemistry. 1-3 million reads were allocated to each sample, representing 1/2000 coverage of

320 human genome.

321

\section{Enzymatic digestion of captured ussDNA}

323 ussDNA extracted from RBC or plasma were treated with dsDNase (Thermal Fisher, \#EN0771) to

324 specifically digest double-stranded DNA and DNase I (NEB, \#M0303S) to digest both double-

325 stranded DNA as well as single-stranded DNA. Specifically, after binding captured ussDNA to

326 streptavidin-coated magnetic beads, the beads were washed to remove biofluids and tissue

327 debris. Washed streptavidin beads were pelleted using a magnetic beads and wash buffer 
328 discarded. $2 \mu \mathrm{L}$ of enzyme, $5 \mu \mathrm{L}$ of $10 \mathrm{x}$ buffer and $43 \mu \mathrm{L}$ of water were mixed and used to suspend

329 DNA-bound streptavidin beads. The mixture was incubated at $37^{\circ} \mathrm{C}$ for $5 \mathrm{~min}$ for dsDNase

330 treatment or at $37^{\circ} \mathrm{C}$ for $10 \mathrm{~min}$ for DNase treatment incubate. Following enzymatic digestion,

331 beads were pelleted again and washed once in $200 \mu \mathrm{L}$ of $10 \mathrm{mM}$ Tris- $\mathrm{HCl}, 1 \mathrm{mM}$ EDTA, $0.05 \%$

332 Tween-20, $100 \mathrm{mM} \mathrm{NaCl}, 0.5 \%$ SDS, and another time in $200 \mu \mathrm{L}$ of $10 \mathrm{mM}$ Tris- $\mathrm{HCl}, 1 \mathrm{mM}$ EDTA,

$3330.05 \%$ Tween-20, $100 \mathrm{mM} \mathrm{NaCl}$. DNA molecules were then released from beads by heat

334 incubation at $95^{\circ} \mathrm{C}$ for $5 \mathrm{~min}$ in $0.1 \times \mathrm{TE}$ buffer.

\section{Reference material preparation}

337 Reference materials were synthetic short single-stranded oligos purchased from Integrated DNA

338 Technologies (IDT). Sequences of reference materials are generated by a custom random

339 generator with GC\% range between $40 \%$ and $60 \%$ and sequence complexity resemble biological

340 sequences. Homology test was implemented to ensure that the sequences did not align to human

341 genome. Single-stranded spike-in references are composed of 24 different oligos at equal

342 concentrations with sizes of $20 \mathrm{nt}, 30 \mathrm{nt}, 40 \mathrm{nt}, 50 \mathrm{nt}, 60 \mathrm{nt}$ and $70 \mathrm{nt}$, with four distinct sequences

343 at each size. Double-stranded spike-in references are composed of 24 oligo duplexes at equal

344 concentrations with sizes of $25 \mathrm{nt}, 35 \mathrm{nt}, 45 \mathrm{nt}, 55 \mathrm{nt}, 65 \mathrm{nt}$ and $75 \mathrm{nt}$, with four distinct sequences

345 at each size. To form oligo duplexes, each two complementary oligos were annealed at $10 \mu \mathrm{M}$ in

346 1x PBS buffer to form a single double-stranded species using a thermal annealing program of

347 denaturing at $95^{\circ} \mathrm{C}$ for $5 \mathrm{~min}$ followed by cooling to $20^{\circ} \mathrm{C}$ at rate of $-0.1^{\circ} \mathrm{C} / 6 \mathrm{~s}$, and stored at $-20^{\circ} \mathrm{C}$ 348 until use. 


\section{ussDNA concentration quantitation}

35124 ssDNA oligos were spiked into hybrid capture mixture (Hyb) at final concentration of

$3521 \mathrm{pM} /$ strand to be used as a reference to estimate concentration of blood-derived ussDNA. Let

$353 R_{\text {genome }}$ denote number of reads aligned to reference genome with size $>20 n t$, and $R_{\text {spikein }}$ denote

354 number of reads aligned to all single-stranded spike-in sequences. The formula for estimating the

355 overall concentration of ussDNA is the following:

356

$$
\text { ussDNA conc. (pM) in Hyb }=\frac{R_{\text {genome }}}{\left(R_{\text {spikein }}\right) / 24}
$$

$$
\text { ussDNA conc. }(\mathrm{pM}) \text { in biofluids }=\frac{R_{\text {genome }} \cdot 2.4}{\left(R_{\text {spikein }}\right) / 24}
$$

* Multiplied by 2.4 because when $100 \mu \mathrm{L}$ of biofluids was added to hybridization mixture, its concentration was diluted by 2.4 -fold.

$$
\text { ussDNA conc. }(\mathrm{ng} / \mathrm{mL}) \text { in biofluids }=\frac{R_{\text {genome }} \cdot 2.4}{\left(R_{\text {spikein }}\right) / 24} \cdot(\text { median length }) \cdot 330 \cdot 10^{-6}
$$

\section{1}

\section{Bioinformatics analysis}

362 Fastq files were pre-processed using custom python scripts as follows. Fastq sequences are

363 trimmed off of adapter sequences, and then quality filtered by retaining reads with greater than

$36480 \%$ of high quality (Q30) bases. Reads with sizes less than 4 bases are considered adapter dimers

365 and was removed from further analysis. Short sequences (<150nt) are end-to-end paired and

366 bases with higher quality between read1 and read2 are retained. Longer reads

367 (150nt<length<290nt) cannot be length-resolved by a single read and thus match of terminal

368 sequences of read1 and read2 is performed to merged matched reads. Next, filtered fastq reads

369 are aligned to reference genome using Bowtie2 to generate sam files containing alignment 
370 information (Fig. S1e). Then custom MATLAB scripts were used to analyze sequence size and

371 distribution.

372

\section{Genome distribution analysis}

374 Genome distribution analysis is based on genomic alignment coordinates given by sam files. For 375 global genome distribution analysis, the GRCh38 genome assembly was separated into bins of $37610,000 \mathrm{bp}$, and the number of reads aligned to each bin was calculated and the coverage was 377 plotted as a heatmap cross all chromosomes (excluding Y chromosome). Chromosome bias was 378 analyzed by counting reads aligned to each chromosome including the mitochondrial genome

379 (chrM). The chromosomal density was then calculated by dividing aligned reads by chromosome 380 size, which was then normalized to global density. Genomic locations of functional elements 381 including gene coding regions, introns, telomeres, promoters and enhancers were downloaded 382 from UCSC genome browser. Here, telomeres are defined as most distal 1M bases flanking all 383 chromosomes. Reads with overlapping coordinates were considered the corresponding 384 functional element. Statistical analysis of the distribution of functional elements assumes that 385 the reads aligned to each element is proportional to the element size. Each read was considered 386 a trial in binomial distribution with the probability being the fraction of genome occupied by 387 certain element. Therefore, for each functional element, significance level could be calculated 388 from binomial distribution with probability and aligned reads. 
392 The data supporting the findings of this study are available within the paper. Source data are 393 available upon request.

395 Code availability

396 The custom Python and MATLAB scripts used in this study are available as Supplementary

397 Software.

398

399 Acknowledgements

400 This work was supported by NIH grant no. R01CA233364 to D.Y.Z. We thank Dr. Ping Song for 401 supervising phlebotomy practice.

402

403 Competing interests

404 L.Y.C., L.R.W. and P.D. declare competing interests in the form of consulting for Nuprobe USA.

405 D.Y.Z. declares a competing interest in the form of consulting for and equity ownership in 406 Nuprobe USA, Torus Biosystems and Pana Bio.

407

408 


\section{References}

410

411 1. Snyder, M.W., Kircher, M., Hill, A.J., Daza, R.M. \& Shendure, J. Cell-free DNA comprises an in vivo nucleosome footprint that informs its tissues-of-origin. Cell 164, 57-68 (2016).

$4132 . \quad$ Lo, Y.D. et al. Maternal plasma DNA sequencing reveals the genome-wide genetic and mutational profile of the fetus. Science translational medicine 2, 61ra91-61ra91 (2010).

415 3. Wyatt, A.W. et al. Concordance of circulating tumor DNA and matched metastatic tissue biopsy in prostate cancer. JNCl: Journal of the National Cancer Institute 109 (2017).

417 4. Chae, Y.K. et al. Concordance between genomic alterations assessed by next-generation sequencing in tumor tissue or circulating cell-free DNA. Oncotarget 7, 65364 (2016).

419 5. Szpechcinski, A. et al. Cell-free DNA levels in plasma of patients with non-small-cell lung cancer and inflammatory lung disease. British journal of cancer 113, 476-483 (2015).

421 6. Meddeb, R. et al. Quantifying circulating cell-free DNA in humans. Scientific reports 9, 116 (2019).

423 7. Mouliere, F. et al. Enhanced detection of circulating tumor DNA by fragment size analysis. Science translational medicine 10 (2018).

425 8. Liu, X. et al. Enrichment of short mutant cell-free DNA fragments enhanced detection of pancreatic cancer. EBioMedicine 41, 345-356 (2019).

427 9. Streubel, A. et al. Comparison of different semi-automated cfDNA extraction methods in combination with UMI-based targeted sequencing. Oncotarget 10, 5690 (2019). 
429 10. Cook, L. et al. Does size matter? Comparison of extraction yields for different-sized DNA fragments by seven different routine and four new circulating cell-free extraction methods. Journal of clinical microbiology 56, e01061-01018 (2018).

432 11. Kierzek, E. et al. The influence of locked nucleic acid residues on the thermodynamic properties of 2'-O-methyl RNA/RNA heteroduplexes. Nucleic acids research 33, 50825093 (2005).

435 12. Troll, C.J. et al. A ligation-based single-stranded library preparation method to analyze cell-free DNA and synthetic oligos. BMC genomics 20, 1-14 (2019).

13. Zhang, J.X. et al. Predicting DNA hybridization kinetics from sequence. Nature chemistry 10, $91-98$ (2018).

14. Wu, L.R., Chen, S.X., Wu, Y., Patel, A.A. \& Zhang, D.Y. Multiplexed enrichment of rare DNA variants via sequence-selective and temperature-robust amplification. Nature biomedical engineering 1, 714-723 (2017).

442 15. Jiang, P. et al. Lengthening and shortening of plasma DNA in hepatocellular carcinoma patients. Proceedings of the National Academy of Sciences 112, E1317-E1325 (2015).

444 16. Kelly, R.D., Mahmud, A., McKenzie, M., Trounce, I.A. \& St John, J.C. Mitochondrial DNA copy number is regulated in a tissue specific manner by DNA methylation of the nuclearencoded DNA polymerase gamma A. Nucleic acids research 40, 10124-10138 (2012). 